\title{
THE ORDINARY QUATERNIONS OVER A PYTHAGOREAN FIELD ${ }^{1}$
}

\author{
BURTON FEIN AND MURRAY SCHACHER
}

\begin{abstract}
Let $L$ be a proper finite Galois extension of a field $K$ and let $D$ be a division algebra with center $K$. If every subfield of $D$ properly containing $K$ contains a $K$-isomorphic copy of $L$, it is shown that $K$ must be Pythagorean, $L \cong K(\sqrt{-1})$, and $D$ is the ordinary quaternions over $K$. If one assumes only that every maximal subfield of $D$ contains a $K$ isomorphic copy of $L$, then, under the assumption that $[D: K]$ is finite, it is shown that $K$ is Pythagorean, $L=K(\sqrt{-1})$, and $D$ contains the ordinary quaternions over $K$.
\end{abstract}

Let $K$ be a field and $L$ a finite-dimensional Galois extension of $K$. Suppose $D$ is a division algebra with center $K$ having the property that every maximal subfield of $D$ contains a $K$-isomorphic copy of $L$. We ask what can be concluded about $D, K$, and $L$. In [1] Herstein considered the case where $L$ is quadratic over $K$; he concluded then that $K$ is Pythagorean, $L=K(\sqrt{-1})$, and $D \supset Q_{K}$, the ordinary quaternion algebra over $K$. A Pythagorean field is a field which is formally real in which every sum of squares is a square. The ordinary quaternion algebra $Q_{K}$ is the $K$ algebra $K+K i+K j+K k$ subject to the relations: $i^{2}=j^{2}=k^{2}=-1, i j=-j i=k, j k=-k j=i, k i=-i k$ $=j$.

In this paper we prove the following two theorems, both of which should be viewed as generalizations of [1].

THeOREM 1. Let $L$ be a proper finite Galois extension of $K$ and let $D$ be $a$ division algebra with center $K, Q \neq K$. Suppose that every subfield of $D$ properly containing $K$ contains a $K$-isomorphic copy of $L$. Then $K$ is Pythagorean, $L=K(\sqrt{-1})$, and $D$ is the ordinary quaternion algebra $Q_{K}$.

THEOREM 2. Let $L$ be a proper finite Galois extension of $K$ and let $D$ be $a$ finite-dimensional division algebra with center $K, D \neq K$. Suppose that every maximal subfield of $D$ contains a $K$-isomorphic copy of $L$. Then $K$ is Pythagorean, $L=K(\sqrt{-1})$, and $D$ contains the ordinary quaternions over $K$.

Before proving these results we need a lemma which is presumably well known, but for which we have not been able to find a convenient reference.

Received by the editors October 13, 1975.

AMS (MOS) subject classifications (1970). Primary 16A40.

Key words and phrases. Division algebra, Pythagorean field.

${ }^{1}$ The work of the first author was supported in part by NSF Grant MPS71-02969 while the work of the second author was supported by NSF Grant MPS71-2884. During much of this work the second author was a research fellow at the University of Pisa. He would like to thank the Italian National Research Council (CNR) for their support. 
Lemma 1. Let $K$ be a field. Then $K$ is Pythagorean if and only if $Q_{K}$ is a division algebra and every maximal subfield of $Q_{K}$ is $K$-isomorphic to $K(\sqrt{-1})$.

Suppose $K$ is Pythagorean. Then $Q_{K}$ is a division algebra since -1 is not a sum of two squares in $K$. Let $K(\sqrt{t})$ be a maximal subfield of $Q_{K}$. Then $K(\sqrt{t})$ splits $Q_{K}$ and so -1 is a sum of two squares in $K(\sqrt{t})$. Thus $-1=(a+b \sqrt{t})^{2}$ where $a, b, c, d, t \in K, \sqrt{t} \notin K$. Expanding and using the Pythagorean property of $K$ we have $-1=u^{2}+u^{2} t, u, v \in K$. Thus $t=$ $-w^{2}, w \in K$, so $K(\sqrt{t})=K(\sqrt{-1})$ as desired. Conversely, assume $Q_{K}$ is a division algebra having, up to isomorphism, a unique maximal subfield. Let $t \in K$. Then since

$$
-1=-1-t^{2}+t^{2}=\left(\sqrt{-\left(1+t^{2}\right)}\right)^{2}+t^{2}
$$

$K\left(\sqrt{-\left(1+t^{2}\right)}\right)$ splits $Q_{K}$. Thus $K\left(\sqrt{-\left(1+t^{2}\right)}\right)$ is a maximal subfield of $Q_{K}$ and so $K\left(\sqrt{-\left(1+t^{2}\right)}\right)=K(\sqrt{-1})$. This implies that $1+t^{2}$ is a square in $K$ for all $t \in K$. Thus if $u, v$ are nonzero elements of $K$ then $u^{2}+v^{2}$ $=u^{2}\left(1+v^{2} / u^{2}\right)$ is a square in $K$. It follows that $K$ must be Pythagorean since $Q_{K}$ is a division algebra.

We now turn to the theorems.

Proof of Theorem 1. Since every subfield of $D$ containing $K$ contains a $K$-isomorphic copy of $L$, we must have $[L: K]=p$, a prime. Let $\alpha \in D$ such that $K(\alpha)$ is $K$-isomorphic to $L$ and let $\sigma$ generate the Galois group of $K(\alpha)$ over $K$. By the Skolem-Noether theorem [2, Theorem 4.3.1, p. 99], there is a $\delta \in D$ such that $\delta^{-1} \alpha \delta=\sigma(\alpha)$. Since $L \neq K, \sigma(\alpha) \neq \alpha$. We write $\operatorname{Irr}(\alpha, K)$ for the irreducible polynomial of $\alpha$ over $K$. Since $[K(\alpha): K]=p, \delta^{p}$ commutes with $\alpha$. If $\delta^{p} \notin K$, then $K\left(\delta^{p}\right)$ contains a $K$-isomorphic copy of $L$ and so $\operatorname{Irr}(\alpha, K)$ splits into linear factors in $K\left(\delta^{p}\right)$. Since $K\left(\alpha, \delta^{p}\right)$ is a field and $\alpha$ is a root of $\operatorname{Irr}(\alpha, K)$ in $K\left(\alpha, \delta^{p}\right)$ we must have $\alpha \in K\left(\delta^{p}\right)$. But $K\left(\delta^{p}\right)$ $\subset K(\delta)$ so $\alpha \in K(\delta)$ and $\delta^{-1} \alpha \delta=\alpha$, a contradiction. Thus $\delta^{p} \in K$. Let $D_{0}=\left\{\sum_{i=0}^{p-1} a_{i} \delta^{i} \mid a_{i} \in K(\alpha)\right\}$. Then $D_{0} \subset D$ and $D_{0}$ is $K$-isomorphic to the cyclic algebra $\left(K(\alpha) / K, \sigma, \delta^{p}\right)$.

Let $C_{D}\left(D_{0}\right)$ denote the centralizer in $D$ of $D_{0}$. By [2, Theorem 4.4.2, p. 112], $D \cong D_{0} \otimes_{K} C_{D}\left(D_{0}\right)$. If $C_{D}\left(D_{0}\right) \neq K$, then $C_{D}\left(D_{0}\right)$ is a nontrivial division ring. Let $E$ be a maximal subfield of $C_{D}\left(D_{0}\right)$. Then $E$ contains a $K$ isomorphic copy of $L$ and so $D \supset K(\alpha) \otimes_{K} L$. Since $K(\alpha) \cong L, D$ $\supset L \otimes_{K} L$. This is a contradiction since $L \otimes_{K} L$ has zero divisors. Thus $C_{D}\left(D_{0}\right)=K$ and so $D=D_{0}$. We have established that $[D: K]=p^{2}$ and $D=\left(K(\alpha) / K, \sigma, \delta^{p}\right)$. In particular, $K(\delta)$ is a maximal subfield of $D$ and so $K(\delta) \cong L$. Since $\delta^{p} \in K$, the characteristic of $K$ cannot be $p$. Since $K(\delta)$ is a Galois extension of $K$ of degree $p$ and $\delta^{p} \in K, K$ must contain a primitive $p$ th root of unity. Also, $\operatorname{Irr}(\delta, K)=x^{p}-\delta^{p}$. Since $K(\alpha)$ is $K$-isomorphic to $K(\delta)$, some element of $K(\alpha)$ is a root of $\operatorname{Irr}(\delta, K)$. We clearly may assume that this element is $\alpha$ and so $\alpha^{p}=\delta^{p}$. Since $\delta^{-1} \alpha \delta \in K(\alpha)$ and $\delta^{-1} \alpha \delta$ is a root of $\operatorname{Irr}(\alpha, K)$, we must have $\delta^{-1} \alpha \delta=\zeta \alpha$ where $\zeta$ is a primitive $p$ th root of unity in $K$. An easy induction proves that $\left(\alpha \delta^{-1}\right)^{n}=\zeta^{n(n-1) / 2} \alpha^{n} \delta^{-n}$ for 
$n \geqslant 1$. If $p$ is odd, then $\zeta^{p(p-1) / 2}=1$ and so $\left(\alpha \delta^{-1}\right)^{p}=\alpha^{p} \delta^{-p}=1$. Since $K$ contains all $p$ th roots of unity, $\alpha \delta^{-1} \in K$ and so $\alpha \delta=\delta \alpha$. This is a contradiction and so $p=2$. In this case we have $\left(\alpha \delta^{-1}\right)^{2}=-\alpha^{2} \delta^{-2}=-1$. Since $\alpha \delta^{-1} \notin K, K(\sqrt{-1})$ is a subfield of $D$ and so $L \cong K(\sqrt{-1})$. Without loss of generality we may assume that $\alpha^{2}=-1$ and so $\delta^{2}=-1$. Since $\left(\alpha \delta^{-1}\right)^{2}=$ $-1, \alpha \delta^{-1}=-\delta^{-1} \alpha$ and so $D$ is the ordinary quaternions over $K$. Finally, since all maximal subfields of $D$ are $K$-isomorphic to $L \cong K(\sqrt{-1}), K$ is Pythagorean by the lemma. This proves Theorem 1 .

Proof OF THEOREM 2. If every subfield of $D$ properly containing $K$ contains a $K$-isomorphic copy of $L$, we are finished by Theorem 1 . Assume that $E$ is a subfield of $D, E$, properly containing $K$, and $E$ is maximal with respect to not containing a $K$-isomorphic copy of $L$. $E$ exists because $[D: K]$ is finite. Then $C_{D}(E)$ satisfies the hypotheses of Theorem 1 and so $E$ is Pythagorean, $E L=E(\sqrt{-1})$, and $C_{D}(E)$ is the ordinary quaternions over $E, C_{D}(E)=Q_{E}$. Since $Q_{E} \cong Q_{Q} \otimes_{Q} E, Q_{E} \supset Q_{Q} \otimes_{Q} K=Q_{k}$ and so $D \supset$ $Q_{K} . K$ is formally real since $E$ is. Suppose $L \not K(\sqrt{-1})$. Then take $F$ a subfield of $D, F \supset K(\sqrt{-1}), F$ maximal with respect to not containing a $K$-isomorphic copy of $L$. Then $C_{D}(F)$ satisfies the hypotheses of Theorem 1 so $C_{D}(F)=Q_{F}$. But $\sqrt{-1} \in F$ so $Q_{F}$ has zero divisors. Thus $C_{D}(F)$ has zero divisors, a contradiction. It follows that $L \cong K(\sqrt{-1})$. Finally, we must show that $K$ is Pythagorean. In view of the results already obtained and the lemma, we need only show that every maximal subfield of $Q_{K}$ is $K$-isomorphic to $K(\sqrt{-1})$. Let $V$ be a maximal subfield of $Q_{K}$. If $V ¥ K(\sqrt{-1})$ we may take $W$ a subfield of $D, W \supset V, W$ maximal with respect to $\sqrt{-1} \notin W$. Since $L \cong K(\sqrt{-1}) C_{D}(W)$ satisfies the hypotheses of Theorem 1 so $C_{D}(W)=Q_{W}$. But $W \supset V$ and $V$ splits $Q_{K}$ so $Q_{W}$ has zero divisors. This contradiction completes the proof of Theorem 2.

The following corollary is immediate from Theorem 1.

COROLlaRy 3. Let $D$ be a division algebra finite dimensional over its center $K$. If all maximal subfields of $D$ are Galois over $K$ and are $K$-isomorphic, then $K$ is Pythagorean and $D$ is the ordinary quaternions over $K$.

There are some natural questions open to generalization concerning the results above. Among these the most tantalizing seem to be:

(1) Can the assumption of normality of $L$ in Theorems 1 and 2 be eliminated?

(2) Can the assumption of finite-dimensionality be eliminated from Theorem 2?

\section{REFERENCES}

1. I. N. Herstein, On a theorem of Albert, Scripta Math. 29 (1972), 391-394.

2. , Noncommutative rings, Carus Math. Monographs, vol. 15, Math. Assoc. Amer., distributed by Wiley, New York, 1968. MR 37 \#2790.

Department of Mathematics, Oregon State University, Corvallis, Oregon 97331

Department of Mathematics, University of California, los Angeles, California 90024 